\title{
LETIER
}

\section{Identification of 4370 Expressed Sequence Tags from a 3'-End-Specific cDNA Library of Human Skeletal Muscle by DNA Sequencing and Filter Hybridization}

\author{
G. Lanfranchi, T. Muraro, F. Caldara, B. Pacchioni, A. Pallavicini, \\ D. Pandolfo, S. Toppo, S. Trevisan, S. Scarso, and G. Valle'
}

Centro di Ricerca Interdipartimentale per le Biotecnologie Innovative (CRIBI) Biotechnology Centre, Università degli Studi di Padova, 35121 Padova, Italy

\begin{abstract}
A systematic study on the mRNA species expressed in the human skeletal muscle is presented in this paper. To carry on this study, a new method has been developed for the construction of unbiased cDNA libraries specially designed for the production of ESTs corresponding to the 3 '-end portion of the mRNAs. The method has been applied to human skeletal muscle, where the analysis of the transcription profile is particularly difficult for the presence of several very abundant transcripts. To detect and quantify high-level mRNAs, the first 1054 ESTs were obtained from randomly selected clones. The 10 most abundant transcripts accounted for $>45 \%$ of the clones. Subsequently, these transcripts were identified by filter hybridization, thus making DNA sequencing more productive. Overall, 4370 clones were identified: 3372 by DNA sequencing and 998 by filter hybridization. The number of groups of sequences identifying individual transcripts was relatively low compared with other tissues, resulting in a total of 934 groups out of 4370 ESTs. Of these, 719 groups were represented by only one sequence.
\end{abstract}

The identification of human genes by systematic sequencing of genomic DNA is hindered by the dispersion of the genes among large noncoding regions and by the presence of introns within genes. Systematic sequencing of cDNA libraries is an alternative approach that offers several advantages: First, it should allow the identification of most human genes within a reasonable time (Adams et al. 1991; Sikela and Auffrey 1993); second, cDNA libraries can be prepared from different human tissues, allowing the construction of maps of tissue-specific and stage-specific genes (Okubo et al. 1992; Adams et al. 1993a); furthermore, in some cases the frequency of a given sequence in the cDNA library can be related to the relative abundance of the corresponding mRNA, giving an indication of the level of gene expression (Okubo et al. 1992).

The general strategy of such studies is based on the analysis by a "single pass" systematic se-

\footnotetext{
${ }^{1}$ Corresponding author.

E-MAIL valle@eos.bio.unipd.it; FAX + 39-49-8276280.
}

quencing of random cDNA clones, which results in the production of short partial sequences, generally referred to as expressed sequence tags (ESTs). Several laboratories have recently produced many thousands ESTs from different human tissues and cell lines (Adams et al. 1991, 1992, 1993a,b; Gieser and Swaroop 1992; Okubo et al. 1992; Sikela and Auffrey 1993; Takeda et al. 1993; Liew et al. 1994; Frigerio et al. 1995) and from other organisms (Waterston et al. 1992; Höfte et al. 1993; Wan Kim et al. 1993).

Most protocols result in the production of ESTs that correspond to random positions within the original mRNAs, thus allowing the assembly of contigs belonging to the same mRNA and the definition of full-length transcripts. This is done either by random priming (Adams et al. 1991; Wan Kim et al. 1993) or by oligo(dT) priming followed by directional sequencing from the $5^{\prime}$ end (Adams et al. 1993b). However, owing to the different abundance of the mRNAs, some sequences will be done many times, whereas the uncommon sequences will remain identified only by partial tags or will not be identified at all. 


\section{LANFRANCHI ET AL.}

This is particularly critical in tissues like skeletal muscle, where some mRNAs are known to be expressed at very high levels. Therefore, it is not surprising that most human ESTs currently available in GenBank were obtained from tissues displaying a broad range of transcripts such as brain, liver, and tumor cells. However, a considerable number of ESTs from skeletal muscle have been produced (Sikela and Auffrey 1993), but there is little accompanying documentation.

For an investigation on tissues like skeletal muscle, it might be advisable to aim first at the construction of a reliable catalog of transcripts and only later to the full characterization of the unknown sequences. In this respect Okubo et al. (1992) proposed a strategy where the main objective of EST sequencing is not the identification of full-length mRNAs but rather the production of a catalog in which each transcript is identified only by its 3' end. Thus, systematic random sequencing can be restricted to a relatively small portion of each mRNA, which avoids dispersion and allows the definition of a more accurate catalog. However, the method of Okubo et al. (1992) requires the presence of particular restriction sites in the $3^{\prime}$-end portion of the mRNAs, which could result in the loss of some transcripts and in the production of biased catalogs.

In this paper we describe a new method for the construction of $3^{\prime}$-end cDNA libraries, specially designed for EST sequencing and for further studies of genome mapping (Wilcox et al. 1991; Khan et al. 1992). Furthermore, this new method was combined with a hybridization procedure for identifying the most abundant $\mathrm{mR}$ NAs, and used for a systematic analysis of transcription in human skeletal muscle.

\section{RESULTS \\ Construction and Verification of the cDNA Library}

A schematic representation of the strategy used for the construction of the cDNA library is shown in Figure 1. A more detailed technical description can be found in Methods.

After the production of the first 1054 ESTs, the 10 most abundant transcripts were analyzed to verify the percentage of the cDNA clones that were actually corresponding to the 3 '-end portions of the mRNAs. The large majority (96\%) of

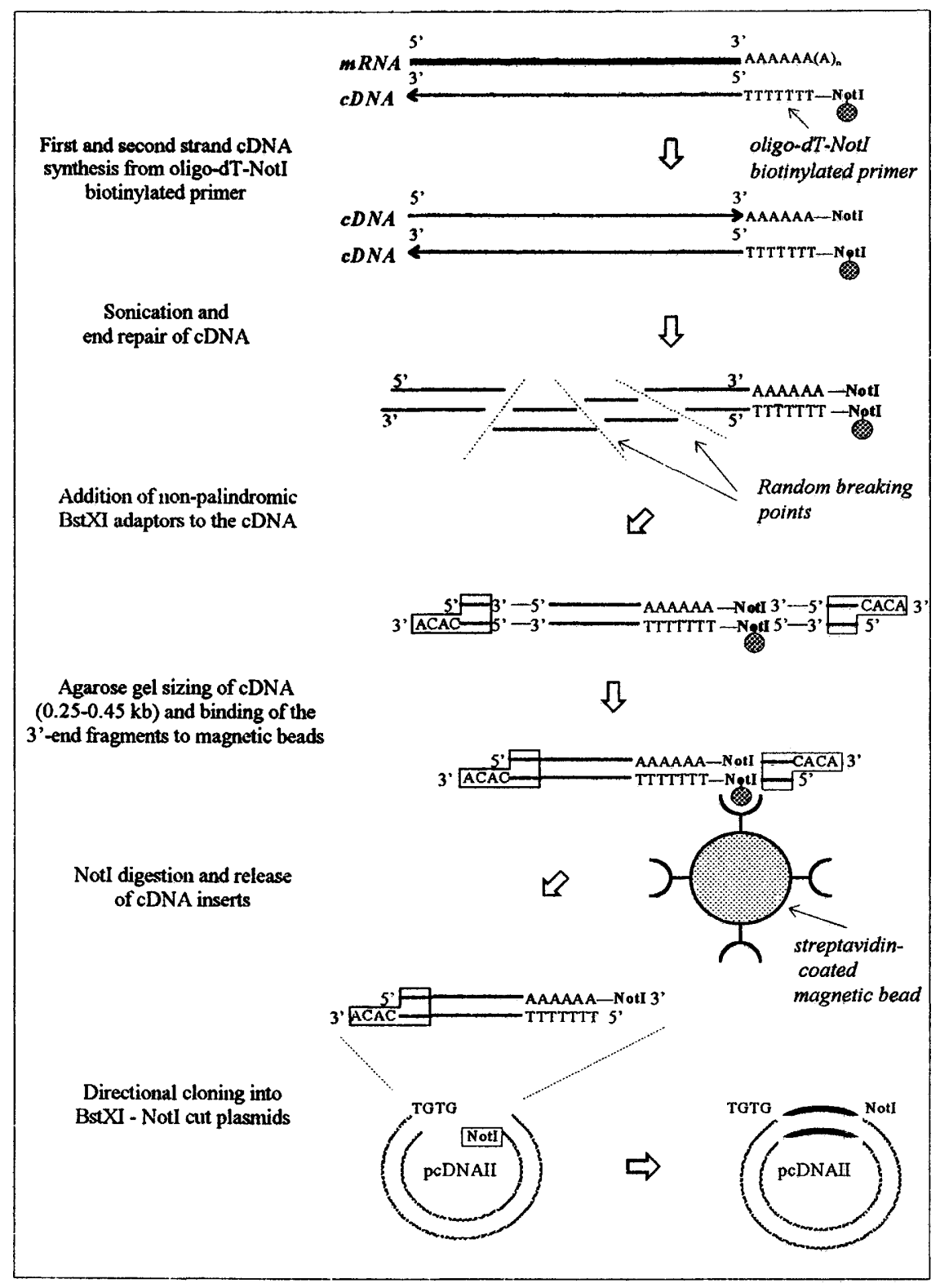

Figure 1 Schematic diagram of the method used for the construction of the CDNA library. More details can be found in the text. 
A CAIALOG OF ESIs FROM HUMAN SKELETAL MUSCLE

the sequences matched to the putative $3^{\prime}$ end of the corresponding mRNA with an approximation of $<10$ bases. Most of the remaining sequences did not reach the $3^{\prime}$ end owing to a premature stop of the electrophoretic run, resulting in termination within 100 bases from the $3^{\prime}$ end. Only $0.8 \%$ of the cDNA inserts, all corresponding to mitochondrial transcripts, did not match the orthodox 3' end of the mRNA. However, these oddterminating sequences could be attributable to variable ends of the mRNAs after processing of the polycistronic mitochondrial transcript rather than to a cloning artifact.

To verify whether the method described in Figure 1 produces cDNA clones with a frequency related to the abundance of the corresponding mRNAs, we performed a Northern blot analysis on five transcripts found at different frequencies in our EST catalog. The results are shown in Figure 2 and confirm the validity of the method.

\section{Computer Analysis and Construction of a Catalog of Transcripts}

Following the criteria described in Methods, the 4370 ESTs were analyzed, and as a result, they could be arranged into 934 groups, each corresponding to a different putative transcript. The 215 transcripts that were found at least twice are listed in Figure 3, where the best similarities to already known sequences are also indicated. As can be seen, most of them correspond to known genes. However, the relative frequency of new genes is much higher in the 719 sequences that were found only once and that account for 146 sequences corresponding to already known genes, 103 corresponding to ESTs already present in GenBank, 273 showing some similarity to already known sequences (68 to ESTs, 82 to Alu elements, and 123 to other genes), and 197 with no significant similarity to any sequence of GenBank.

Overall, the 934 groups correspond to 284 known human sequences, 133 ESTs that were already present in GenBank, 296 sequences showing some similarity to already known sequences (72 to ESTs, 86 to Alu elements, and 138 to other genes), and 221 with no significant similarity to any sequence of GenBank.

\section{Occurrences of Known Transcripts and Comparison with Other EST Catalogs}

One of the most noticeable features of our EST catalog is that mitochondrial transcripts are ex-

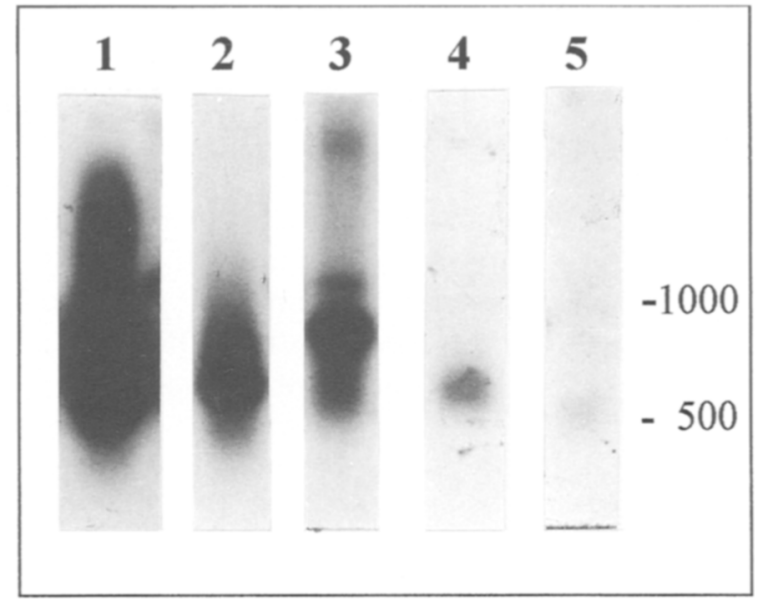

Figure 2 Northern blot analysis of five mRNAs that were found with different abundance in our EST catalog. The five lanes were loaded with the same amount of human skeletal muscle mRNA and were hybridized with the following probes: (Lane 1) ND-3, found in our catalog 443 times; (lane 2) $\alpha$-globin, found 112 times; (lane 3) myosin LC-2, found 91 times; (lane 4) transcript 387, found 3 times; (lane 5) transcript 545, found 2 times. Although the autoradiography does not allow a precise quantitation owing to saturation of the film, it can be easily appreciated that there is a very good correlation between the intensity of the bands and the relative abundance of the ESTs. The length (bases) of the markers is indicated at right.

tremely abundant. They account for 1082 ESTs $(24.8 \%)$, all but one corresponding to the $\mathrm{H}$-strand of the mitochondrial DNA; the exception being an EST of cytochrome oxidase I, that most probably entered into the vector in the wrong orientation.

It was particularly interesting to observe that mitochondrial transcripts behave quite differently from nuclear transcripts (Attardi and Schatz 1988 ) as the $3^{\prime}$ polyadenylated ends often occur at unexpected locations. This is particularly true for certain regions of the mitochondrial DNA. For instance, the ND-4 gene was detected 102 times, 25 of which terminate at variable early positions along the gene. Many ESTs also match the mitochondrial rRNA region (group HSM00007 of Fig. 3 ) and randomly terminate within a wide range of 2600 bases, always corresponding to the $\mathrm{H}$-strand of the mitochondrial genome. Because most of these odd-terminating RNAs have long poly(A) stretches at their $3^{\prime}$ end, it is conceivable that they actually correspond to real RNA terminations. 
LANFRANCHI ET AL.

The catalog of transcripts from human skeletal muscle was compared with other EST catalogs such as heart (Liew et al. 1994), fetal brain (Adams et al. 1993b), hippocampus (Adams et al. 1993b), pancreatic islet cells (Takeda et al. 1993), and HepG2 cells (Okubo et al. 1992). The comparison is quite interesting and indicative of major differences; however, it should be taken into account that in all these projects the cDNA libraries were produced by a variety of methods, often aiming at different goals. However, in all the above catalogs, the frequency of mitochondrial ESTs is much lower: $12 \%$ in heart, $10.3 \%$ in hippocampus, $1.7 \%$ in fetal brain, $1 \%$ in pancreatic islet cells, and $<0.2 \%$ in HepG 2 cells.

As expected, transcripts specific for contractile elements are also very frequent in our EST catalog, in particular ( $\alpha$-actin that was found 373 times $(8.5 \%)$. This abundance is remarkably high compared with heart, where no nuclear transcripts were found to be $>1 \%$ of the total, the two most abundant being ( $\beta$-myosin $\mathrm{H}$-chain and $(\alpha-$ tropomyosin $(0.87 \%$ and $0.42 \%$, respectively).

The frequency of transcripts involved in protein synthesis is also relatively high, with 199 ESTs (4.5\%) matching mRNAs for ribosomal proteins. This percentage is higher than that found in most other tissues.

\section{DISCUSSION}

Libraries of short cDNA fragments corresponding to the 3 -end regions of the mRNAs have the inconvenience of not being very informative in terms of coding sequence; however, they offer several advantages. First, the resulting sequencing projects are restricted to a relatively small region of the mRNAs allowing a better and faster

Figure 3 Catalog of transcripts, obtained from the analysis of 4370 ESTs. A total number of 934 distinct sequences were identified, each corresponding to a different transcript. However, only the 215 transcripts with at least two ESTs are listed in the catalog, leaving out the 719 that occurred only once. The catalog is ordered according to the times that each transcript was found (column 2). The first column indicates the code of the transcript. The best similarity found with sequences in GenBank or SWISS-PROT data bases, with the corresponding descriptions are reported in columns 3 and 4 . The asterisks $\left(^{*}\right)$ indicate the 10 sequences that were also identified by dot-blot hybridization. compilation of catalogs of transcripts (Okubo et al. 1992). Second, all the mRNAs, regardless of their length, have an equal opportunity of being

\begin{tabular}{|c|c|c|c|}
\hline Code & Times & Best similarity & Description \\
\hline ISM00002 & $443^{*}$ & MIHSGENOM & NADH dehydrogenase chain 3 \\
\hline HSM00026 & $373 *$ & HUMSAACT & Skeletal alpha-actin \\
\hline HSM00012 & $319^{*}$ & MIHSGENOM & ATPase 6 \\
\hline HSM00007 & 278 & MIHSGENOM & Mit.-DNA (1-2600; H strand) \\
\hline HSM00008 & $195^{*}$ & MIHSGENOM & Cytochrome C oxydase II \\
\hline HSM00018 & $157^{*}$ & MIHSGENOM & NADH delhydrogenuse chain 2 \\
\hline HSM00028 & $137^{*}$ & MIHSGENOM & Cytochrome C oxydase III \\
\hline HSM00017 & $112 *$ & HSAGLO1 & Alpha globin \\
\hline HSM00049 & 102 & MIHSGENOM & NADH dehydrogenase chains $4-4 \mathrm{~L}$ \\
\hline HSM00014 & $91^{*}$ & HSMYLC2 & Myosin LC-2 \\
\hline HSM00030 & $87^{*}$ & HUMTNTS & Slow skeletal muscle troponin $\mathrm{T}$ \\
\hline HSM00009 & $79 *$ & HUMCKMM8 & Creatine kinase (CKMM) \\
\hline HSM00050 & 51 & MIHSGENOM & Cytochrome b \\
\hline HSM00013 & 46 & HUMGAPDH & Glyceraldehyde-3-P dehydrogen. \\
\hline HSM00034 & 45 & MIHSGENOM & Cytochrome $\mathrm{C}$ oxydase I \\
\hline HSM00258 & 41 & MIHSGENOM & NADH dehydrogenase chain 1 \\
\hline HSM00064 & 37 & HUMTROPI07 & Troponin I \\
\hline HSM00015 & 33 & & - NEW SEQUENCE - \\
\hline HSM00099 & 32 & OCMYLC22 & Similar to rabbit myosin L-2-2 \\
\hline HSM00127 & 30 & HUMALDOAA & Fructose 1,6-diphosphate aldolase A \\
\hline HSM00055 & 26 & HUMTROPSK & Skeletal beta-tropomyosin \\
\hline HSM00461 & 25 & HUMCYTVIIA & Cytochrome c oxidase subunit VIIa \\
\hline HSM00029 & 24 & HSMHCBR & Beta-myosin H-chain (slow) \\
\hline HSM00340 & 23 & HSMG03 & Myoglobin ex \\
\hline HSM00178 & 21 & HSRPL41 & Human homologue to yeast $r \mathrm{pL} 41$ \\
\hline HSM00302 & 21 & HUMTROPNIN & Troponin I fast-twitch isoform \\
\hline HSM00743 & 20 & HSR & Ribosomal pS11 \\
\hline HSM00016 & 20 & HUN & Desn \\
\hline HSM00019 & 20 & HSE & (skelet \\
\hline HSMI00060 & 17 & HSF & Fast 2a my \\
\hline HSM00700 & 17 & HSU14973 & Ribosomal \\
\hline HSM00292 & 17 & M2 & Rosenthal fil \\
\hline HSM00451 & 16 & HST & skelet: \\
\hline HSM00187 & 15 & HSRPL37A & Ribosomal pL \\
\hline HSM 00023 & 15 & HUMCOXVIM & \\
\hline HSM00778 & 14 & HSLLREP3 & LLRep3, repetitive DNA \\
\hline HSM00512 & 13 & HSBGL1 & Beta-globin \\
\hline HSM00351 & 13 & HSS & rotein C \\
\hline HSMI00105 & 13 & HUMTROPA2 & oponyosin \\
\hline HSM00460 & 12 & $\mathrm{MIH}$ & -14171, H strand) \\
\hline HSM00574 & 11 & HUM & phosphoprotein P1 \\
\hline HSM100100 & 11 & YGM20 & Glycogen p \\
\hline HSM100096 & 10 & HST & ponin $\mathrm{C}(\mathrm{Tn} C)$ \\
\hline HSM00950 & 10 & HSTR & yosin \\
\hline HSM00189 & 10 & HUMPPARP2 & Acidic ribosomal phosphoprotein $\mathrm{P} 2$ \\
\hline HSM00294 & 10 & HUMQM & IVilm's tumor-related prot. QM \\
\hline HSN00899 & 9 & MIHSGENOM & NADH dehydr \\
\hline HSM100124 & 9 & HSTITIN & Titin \\
\hline HSM00264 & 9 & HUMEF2A & Elongation fac \\
\hline HSM00445 & 9 & HUMMLC1 V7 & Ventric. slow \\
\hline HSM 100003 & 9 & HUMSRAA & \\
\hline HSM00341 & 8 & HSENO3BE & Muscle beta-enolase \\
\hline HSM00110 & 8 & HSHSP27L & tein HSP27 \\
\hline HSMO0459 & 8 & HSMYH1R & Myo \\
\hline HSM00128 & 8 & HUMAK1 & Cytosolic adenylate kimase (AK1). \\
\hline HSM00402 & 8 & HUMAMHBA123 & GTP binding prot. related to $\mathrm{MHC}$ \\
\hline HSM00355 & 8 & ISB76II082 & EST (skel. muscle) \\
\hline HSM00577 & 7 & & Ribosomal pl \\
\hline HSM100826 & 7 & HUMCOXCA & Cytochrome coxidase V'B \\
\hline HSM00575 & 7 & HUMIRPL37Z & Ribosomal pL37 \\
\hline HSM00242 & 7 & HUMTCG & D loop region (1-580 11-strand) \\
\hline HSM01684 & 7 & HUMRPS24A & Ribosomal pS24 \\
\hline HSM00848 & 7 & RABATPAD & Similar to rabbit fast skel. ATPas \\
\hline HSM00401 & 6 & HSTPIIG & Triosephosphate isomerase \\
\hline HSM00072 & 6 & HUMCIClA & Cytochrome c-1 \\
\hline
\end{tabular}

Figure 3 (Continued on facing page.) 
A CATALOG OF ESTS FROM HUMAN SKELETAL MUSCLE

\begin{tabular}{|c|c|c|c|c|c|c|c|}
\hline Code & Times & Best similarity & Description & Code & Times & Best similarity & Description \\
\hline ISM00282 & 5 & HSRPS18 & Ribosomal pS18 & HSM01132 & 2 & HSRPS 12 & Ribosomal pS12 \\
\hline ISM01174 & 5 & HUMCH13C4A & Translat. controlled tumor prot. & HSM00781 & 2 & HSRPS8 & Ribosomal pS8 \\
\hline HSM01289 & 5 & HUMRPS21X & Ribosomal pS21 & HSM00610 & 2 & HSU12465 & Ribosomal pL35 \\
\hline ISM00771 & 5 & HUMRPS6 & Ribosomal pS6 & HSM01699 & 2 & HSU14966 & Ribosomal pL5 \\
\hline ASM01257 & 5 & HUMTRT & Skeletal troponin $\mathrm{T}$ isoform & HSM02436 & 2 & HSU14972 & Ribosomal pS10 \\
\hline ISM00686 & 5 & HSB96B062 & EST (skel. muscle) & HSM02522 & 2 & HSU16738 & Clone containing trinucl. repeat \\
\hline HSM00067 & 5 & HSBA7C011 & EST (skel. muscle) & HSM00378 & 2 & HUMALBP & Adipocyte lipid-binding protein \\
\hline HSM01284 & 5 & T07882 & EST (fetal brain) & HSM00557 & 2 & HUMANCDA & Adipsin/complement factor D \\
\hline HSM01705 & 5 & & - NEW SEQUENCE - & HSM02228 & 2 & HUMCD63 I & Lysosomal glycoprotein CD63 \\
\hline HSM01267 & 4 & HS23KDHBP & $23 \mathrm{kD}$ highly basic protein & HSM00125 & 2 & HUMCOX4AA C & Cyt-c oxidase subunit IV \\
\hline ASM01578 & 4 & HSDF1F05 & F1-F0 ATP synthase & HSM00998 & 2 & HUMCPB & Calphobindin II \\
\hline HSM00331 & 4 & HSUBPSE1 & Ubiquitin pseudogene EHB4 & HSM01064 & 2 & HUMCRP04 & Cysteine-rich protein (CRP) \\
\hline HSM00795 & 4 & HUMMLC1SA & Myosin LC-1s (slow) & HSM00417 & 2 & HUMFIXG & Factor $\mathrm{XX}$ antihemophilic factor $\mathrm{B}$ \\
\hline HSM01280 & 4 & HUMMPSI & Metallopanstimulin & HSM02784 & 2 & HUMGLGGA I & Unusual fetal A-gamma-globin \\
\hline HSM00594 & 4 & HUMORFA & Cardiac Autoantigen & HSM00274 & 2 & HUMGLPEX S & Se-dependent glutathione perox. \\
\hline HSM01738 & 4 & MIHSGENOM & Cytochrome $\mathrm{c}$ oxidase subunit I & HSM01760 & 2 & HUMHMGYD I & HMG-Y protein isoform \\
\hline HSM01391 & 4 & HUMGS00977 & EST, Promyelocytes & HSM00581 & 2 & HUMORF & ORF, T-lymphocyte cells \\
\hline HSM01696 & 4 & T31711 & EST S' end (embryo) & HSM00493 & 2 & HUMPAIA & Plasminogen activator inhibitor-1 \\
\hline HSM00760 & 4 & HSBAT2 & Sequence with Alu class A & HSM01105 & 2 & HUMRIBPROC $\mathrm{F}$ & Ribosomal p L11 \\
\hline HSM00245 & 4 & & - NEW SEQUENCE - & HSM02268 & 2 & HUMRIBPROD F & Ribosomal pL18a \\
\hline HSM01423 & 4 & & - NEW SEQUENCE - & HSM00793 & 2 & HUMRPL30A & Ribosomal pL30 \\
\hline HSM01192 & 3 & HSABLGR3 & Proto-oncogene tyrosine kinase & HSM00514 & 2 & HUMRPS13A & Ribosonal pS13 \\
\hline HSM00120 & 3 & HSEF1DELA & Elongation factor-1-delta & HSM01285 & 2 & HUMRPS17 & Ribosomal pS17 \\
\hline HSM01598 & 3 & HSHA44G & Alpha-tubulin, exons 1-3 & HSM02547 & 2 & HUMSET & Set gene (putative oncogene) \\
\hline HSM00349 & 3 & HSIGF27 & Insulin-like growth factor IGF-2 & HSM00094 & 2 & HUMTCBA & TCB (thyroid horm.-bind. prot.) \\
\hline HSM01331 & 3 & HSU02032 & Ribosomal pL23a & HSM00001 & 2 & HUMTCRADCV & T-cell receptor C-alpha V-delta \\
\hline HSM01879 & 3 & HSU12404 & Csa-19 & HSM01306 & 2 & HUMTLCA & ADP/ATP translocase \\
\hline HSM00246 & 3 & HSU14969 & Ribosomal pL28 & HSM01564 & 2 & HUMUBI13 & Ubiquitin \\
\hline HSM00446 & 3 & HSU16660 & Peroxisomal enoyl CoA hydratase & HSM00525 & 2 & S73035S9 & Guanosine-P reductase \\
\hline HSM01116 & 3 & HSUBA52P & Ubiquitin-52 aa fusion protein & HSM01449 & 2 & $\mathrm{HSB} 35 \mathrm{C} 032$ & EST (skeletal muscle) \\
\hline HSM02210 & 3 & HSUBA80R & Uba80 mRNA for ubiquitin & HSM03052 & 2 & HSB95E072 & EST (skeletal muscle) \\
\hline HSM01457 & 3 & HUMFERH & Ferritin $\mathrm{H}$ chain & HSM01240 & 2 & HSDHII070 & EST, (heart) \\
\hline HSM00062 & 3 & HUMFERL & Ferritin L chain & HSM01328 & 2 & M91220 & EST (retinal pigment) \\
\hline HSM01652 & 3 & HUMGSTM4A & Glutathione transferase & HSM00498 & 2 & T03426 & EST (infant brain) \\
\hline HSM00252 & 3 & HUMH19 & H19 RNA gene & HSM02958 & 2 & T05586 & EST (brain) \\
\hline HSM01501 & 3 & HUMMTATP3 & ATP synthase & HSM00746 & 2 & T06948 & EST (fetal brain) \\
\hline HSM100022 & 3 & HUMNRF1A & NRF1 protein & HSM01827 & 2 & T10004 & EST (normalized cDNA; brain) \\
\hline HSM00190 & 3 & HUMRPS20 & Ribosomal pS20 & HSM02603 & 2 & T10156 & EST (normalized cDNA; brain) \\
\hline HSM00299 & 3 & HUMRYR & Ryanodine receptor & HSM01342 & 2 & T11087 & EST (human pancreatic islet) \\
\hline HSM01040 & 3 & HUMSAPC1 & Cerebroside sulfate activator & HSM01483 & 2 & T16091 & EST 3' (infant brain) \\
\hline HSM00338 & 3 & HUMSCAR & Scar protein & HSM02700 & 2 & T24043 & EST 3' (brain) \\
\hline HSM02185 & 3 & HUMTBP1 & Human virus tat-binding protein-1 & HSM02171 & 2 & $\mathrm{~T} 32287$ & EST (brain) \\
\hline HSM02283 & 3 & S42658 & Ribosomal pS3 & HSM02095 & 2 & T34729 & EST (brain) \\
\hline HSM00869 & 3 & HSAFIF085 & EST (fibroblast) & HSM00202 & 2 & S75201 & Sequence with Alu class A \\
\hline HSM01327 & 3 & HUM $0000 \mathrm{~A} 25$ & EST 3 ' end (HepG2 cell line) & HSM00384 & 2 & HSU04737 & Sequence with Alu class C \\
\hline HSM00075 & 3 & T03744 & EST (infant brain) & HSM00759 & 2 & HSRSPAC & Sequence with Alu class E \\
\hline HSM00119 & 3 & T07953 & EST (brain) & HSM02710 & 2 & M62107 & Similar to EST (hippocampus) \\
\hline HSM01519 & 3 & T18875 & EST 5 ' end (testis) & HSM02912 & 2 & $\mathrm{~T} 24920$ & Similar to EST (colorectal cancer) \\
\hline HSM02227 & 3 & T29985 & EST 3 end (adipose tissue) & HSM01782 & 2 & BTCIKFYI & Similar to bovine NADH dehydr. \\
\hline HSM00194 & 3 & T30025 & EST 3 ' end (adipose tissue) & HSM02395 & 2 & BTCIMNLL & Similar to bovine NADH dehydr. \\
\hline HSM01448 & 3 & T30687 & EST 5' end (spleen) & HSM00797 & 2 & CVGEMSZFM : & Similar to vector pGEM-5Zf(-) \\
\hline HSM01092 & 3 & T31434 & EST 5 end (embryo) & HSM01293 & 2 & HS165 & Similar to $165 \mathrm{kD}$ titin-associated \\
\hline HSM00517 & 3 & HSAAACTPB & Similar to EST (placenta) & HSM102386 & 2 & IHSL35A & Similar to ribosomal pL35a \\
\hline HSM01265 & 3 & HSB68E072 & Sinilar to EST (skeletal muscle) & HSM 03020 & 2 & HSU14970 & Similar to ribosomal pS5 \\
\hline HSM00290 & 3 & BOVNADHURD & Similar to bovine NADH delyydr. & HSN101089 & 2 & HUMRPS14 & Similar to ribosomal pS14 \\
\hline HSM00385 & 3 & BTCIB8 & Similar to bovine ubiq. reductase & IISMI00041 & 2 & HUMRPZH 21 & Similar to ribosomal pL44 \\
\hline HSM00138 & 3 & HSRPL19 & Ribosomal pL19 & HSN 01429 & 2 & MIBTCIB22 & Similar to bovine NADH dehydr. \\
\hline HSM00547 & 3 & HUMTMIIE & Similar to epithelial tropomyosin & HSM 00101 & 2 & RATGAP01 & Similar to rat GAP-43 gene \\
\hline HSM00149 & 3 & & - NEW SEQUENCE - & HSN IO0005 & 2 & & - NEW SEQUENCE - \\
\hline HSM00387 & 3 & & - NEW SEQUENCE - & HSM 00150 & 2 & & - NEW SEQUENCE - \\
\hline HSM01533 & 3 & & - NEWV SEQUENCE - & HSM00275 & 2 & & - NEW SEQUENCE - \\
\hline HSM00304 & 2 & HSAT3 & Antithrombin III & HSM00284 & 2 & & - NEW SEQUENCE - \\
\hline HSM00897 & 2 & HSCOVIC & Cytochrome $\mathrm{c}$ oxidase subunit VIc & HSM00288 & 2 & & - NEW SEQUENCE - \\
\hline HSM00838 & 2 & HSCOX7BM & Cytochrome c oxidase (coxVIIb) & HSM00315 & 2 & & - NEW SEQUENCE - \\
\hline HSM02146 & 2 & HSCYCR & T-cell cyclophilin & HSM 100374 & 2 & & - NEW SEQUENCE - \\
\hline HSM01900 & 2 & HSHSC70 & Heat shock cognate protein & HSM00545 & 2 & & - NEW SEQUENCE - \\
\hline HSM02261 & 2 & HSHSP70B & Hent shock protein 70 & HSM00769 & 2 & & - NEW SEQUENCE - \\
\hline HSM01636 & 2 & HSL31 & Ribosomal pL31 & HSN100836 & 2 & & - NEW SEQUENCE - \\
\hline HSN:01987 & 2 & HSLEC14K & Beta-galactoside-binding lectin & HSM00875 & 2 & & - NEW SEQUENCE - \\
\hline HSM00886 & 2 & HSMLC3F & Myosin LC-3f(fast) & HSMO0957 & 2 & & - NEW SEQUENCE - \\
\hline HSM00137 & 2 & HSPAG & Proliferation-associated gene & HSMO0964 & 2 & & - NEW SEQUENCE - \\
\hline HSM100697 & 2 & HSPEABP & Phosphatidylethanolamine binding & HSM101737 & 2 & & - NEW SEQUENCE - \\
\hline HSM00698 & 2 & HSPMIIPR & PMI (putative receptor protein) & HSM01758 & 2 & & - NEW SEQUENCE - \\
\hline HSM02247 & 2 & HSRP26AA & Ribosomal pL26 & HSIIO2190 & 2 & & - NEW SEQUENCE - \\
\hline HSM01235 & 2 & HSRPL38 & Ribosomal L38 & IHSM03043 & 2 & & - NEW SEQUENCE - \\
\hline
\end{tabular}




\section{LANFRANCHI ET AL.}

represented. Third, the cDNA inserts can be sequenced in the direction $5^{\prime} \rightarrow 3^{\prime}$, thus avoiding passing through the poly(A) that generally results in very poor or unreadable DNA sequences.

Our strategy of random fragmentation of the cDNA by sonication, followed by a very stringent selection of the 3'-end fragments, produced very satisfactory results as the great majority of our cDNA inserts were found to match the 3 '-end region of their corresponding mRNAs (see Results) and transcripts such as titin and ryanodine receptor $(14,985$ and 15,345 bases long) were detected nine and three times, respectively, confirming that also very large transcripts could be processed to $3^{\prime}$-end inserts similar to those derived from small transcripts.

A method for the production of 3 -endspecific cDNA libraries had been described previously by Okubo et al. (1992). They made use of restriction enzymes such as $\mathrm{MboI}$, for the double function of fragmenting the cDNA and producing sticky ends useful for directional insertion into the vector. We consider our new protocol for the construction of the cDNA library an improvement on the general strategy, as sonication allows a more random fragmentation of the cDNA than restriction enzymes. For instance, among the 10 most abundant transcripts, 3 do not have an $\mathrm{MboI}$ site (ND-2, Cox-3, and $\alpha$-globin) and 1 (myosin LC-2) has an Mbol site at $<20$ bases from the poly(A); therefore, 4 transcripts amongst the 10 most abundant would not be cloned using that procedure.

A relevant feature of our 3 '-end-restricted cDNA library is that the frequency of an EST gives a good indication of the relative abundance of the corresponding mRNA. This is an important piece of information for expression studies, but it must be considered together with the inconvenience that the most abundant ESTs could be resequenced many times, wasting time and effort. In principle, our method for the production of 3 '-end-restricted cDNA libraries could be used after normalization of the library (Ko 1990; Patanjali et al. 1990), but the quantitative information would be lost. In this work we used the dot-blot hybridization as an alternative approach to overcome the problem of abundant transcripts, while maintaining the quantitative information. This was facilitated by having a cDNA library restricted to only the 3 '-end regions, which allowed an easier construction of specific probes. However, at a later stage of the work, we may consider to continue the search for the rarest transcripts using a normalized library restricted to the 3 '-end regions.

As mentioned in the introductory section, $3^{\prime}$ end ESTs are very suitable for mapping studies (Wilcox et al. 1991; Khan et al. 1992). In this respect, we have started a preliminary project in collaboration with other laboratories, using a panel of rodent/human somatic cell hybrids (Wilcox et al. 1991). We found that in $>90 \%$ of the cases the differences with the rodent sequences were such that a PCR product was only observed from human DNA, making the analytical procedures very simple.

The ESTs described in this paper have been submitted to the EMBL data base (accession nos. F15505-F155554 and F15586-F19692). Further information on this work, including the ABIchromatogram images, are available at the web site http://eos.bio.unipd.it.

\section{METHODS}

\section{Construction of the 3'-End-specific cDNA Library}

A 12-gram sample of human pectoral muscle was obtained from a mastectomy of an adult woman and, after cleaning out the tumor tissue and the infiltrated lymph nodes, it was prepared as described by Chomczynski and Sacchi (1987). Polyadenylated RNA was selected by oligo(dT) column chromatography using standard techniques (Aviv and Leder 1972).

The construction of the cDNA library was based on the Librarian II kit (Invitrogen), with some major changes in the procedure (Fig. 1). The first cDNA strand was synthesized from a specially designed oligo(dT)-NotI primer in which the 5 '-terminal nucleotide was biotinylated. After completion of the second strand, the cDNA was sonicated for $10 \mathrm{sec}$ with a Branson probe set at $100 \mathrm{~W}$. Then cDNA fragments were repaired with T4 DNA polymerase, ligated to $B s t \mathrm{X} 1$ nonpalindromic adaptors, and size-fractionated on low-melting-point agarose (SeaPlaque, FMC) gels. The cDNA fragments of $250-450 \mathrm{bp}$ were extracted with $\beta$-agarase (New England Biolabs), dissolved in TTL buffer [0.3 $\mathrm{M}$ Tris at $\mathrm{pH} 8.0,6 \mathrm{M} \mathrm{LiCl}$, and $0.3 \%(\mathrm{vol} / \mathrm{vol})$ Tween 20$]$, and incubated with $20 \mu \mathrm{l}$ of avidin-coated paramagnetic beads (Dynabeads, Dynal) at $42^{\circ} \mathrm{C}$ for $30 \mathrm{~min}$, with gentle mixing every $5 \mathrm{~min}$. After three washes with $300 \mu \mathrm{l}$ of TTL and two washes with water, the $3^{\prime}$ cDNA ends were released from the beads by NotI digestion. The cDNA was directionally cloned into a BstX1, Notl-digested pcDNAII plasmid (Invitrogen), and used for transformation of the Escherichia coli TOP10F' strain using an electroporator.

\section{DNA Sequencing}

Single bacterial colonies were collected with sterile toothpicks, transferred into $50 \mu \mathrm{l}$ of PCR buffer [ $20 \mathrm{~mm}$ Tris-HCl at $\mathrm{pH} 8.3,50 \mathrm{mM} \mathrm{KCl}, 2 \mathrm{mM} \mathrm{MgCl}_{2}$, and $0.1 \%$ (vol/vol) Tween 20], lysed at $95^{\circ} \mathrm{C}$ for $10 \mathrm{~min}$ in 96-well microtiter 


\section{A CATALOG OF ESIs FROM HUMAN SKELETAL MUSCLE}

plates, and processed as described by Hultman et al. (1991) using paramagnetic beads (Dynal). Single-stranded templates were processed in a sequencing reaction by the dyedeoxy terminator chemistry developed by Applied Biosystems, using a sequencing primer (5'-CTCGGATCCACTAGTAACG-3') located 21 bases upstream from the first nucleotide of the cDNA insert. Sequencing gels were run on Applied Biosystems DNA sequencers.

\section{Northern Blotting}

Skeletal muscle mRNA was denatured with glyoxal-DMSO buffer (McMaster and Carmichael 1977) and separated on agarose gels using $10 \mathrm{~mm}$ sodium phosphate $(\mathrm{pH} 7.0)$ as the running buffer. The mRNA was then transferred to nylon sheets (Hybond $\mathrm{N}+$, Amersham) in $40 \mathrm{~mm} \mathrm{NaOH}$. Probes were obtained by PCR amplification of microlysate colonies with specific internal primers and labeled using the random priming technique (Feinberg and Vogelstein 1983). Standard protocols were used for hybridization and high stringency washing of Northern blots (Thomas 1980).

\section{Preparation of Specific Probes and Dot-blot Analysis}

To allow an easy identification of the most frequent ESTs without the need of DNA sequencing, a filter hybridization procedure (dot-blot) was implemented as a standard step of our protocol after the first 1054 ESTs had already been sequenced. The dot-blot procedure involved the transfer of the amplified cDNA samples from 96- to 384well plates, followed by the spotting of 200-nl aliquots from each sample onto nylon filters (Hybond $\mathrm{N}+$, Amersham) using 384-teeth disposable devices (Genetix). Filters were then processed for hybridization using standard methods. The following 10 sequences were analyzed: ND3 , $\alpha$-actin, ATPase- 6 , Cox-2, ND-2, Cox-3, $\beta$-globin, myo$\sin$ LC-2, troponin T, and creatine kinase, hereafter referred to as abundant ESTs.

Probes specific for the 10 abundant ESTs were obtained by PCR amplification of the regions corresponding to the terminal 200-250 bases at the 3' end of the mRNAs, using specific internal primers. The amplified DNA fragments were cloned into plasmid vectors, and the inserts were purified and labeled using the nonradioactive ECL system (Amersham).

The reliability of this hybridization method was checked by performing a double analysis on a series of 384 samples, both by dot-blot hybridization and by DNA sequencing. The results of this comparison showed that under the stringency conditions used, $-3 \%$ of false negatives resulted from dot-blot hybridization.

Because some ESTs were identified both by dot-blot hybridization and by DNA sequencing, some criteria had to be defined to avoid counting twice the same ESTs. Furthermore, it had to be taken into account that some samples were lost during the procedures for DNA sequencing, whereas the ESTs identified by hybridization did not need any further analysis and could not be lost. Therefore, a simple counting of the samples positive in the dot-blot assay would have led to an overevaluation of those samples. To avoid these problems, the occurrences of the ESTs identified by dot-blot were calculated as follows. First, the percentage of the most frequent ESTs was calculated on the first 1054 samples (i.e., before the filter hybridization procedure was implemented): For instance, $\alpha$-actin was found 90 times and the 10 abundant ESTs together were found 481 times, against 573 other ESTs. Second, it was considered that a total number of 3372 ESTs had been sequenced, of which 996 were abundant and 2376 were other ESTs. Finally, the occurrences of each abundant EST were calculated using the ratio observed in the first 1054 . For instance, the occurrences of $\alpha$-actin resulted $90 \times 2376 / 573=373$ occurrences.

\section{Computer Management of the Data}

Each new EST was first corrected, and any residual sequence of the vector or poly(A) was removed. Each EST was then analyzed with the program FASTA (Pearson and Lipman 1988), against the other ESTs already present in our data base. Two ESTs were considered to match each other when they shared at least 50 bases with at least $98 \%$ identity. For longer sequences, the threshold was decreased $1 \%$ every 25 bases. If with the above criteria a new EST was matching at the same time two different existing groups, then the two groups were joined together. In this case a manual check was performed to verify whether any EST was a recombinant with a double insert. Only two double inserts were identified, which allowed us to estimate the percentage of bad recombinant cDNA clones to $<0.1 \%$.

To attribute to each group a possible identity, each EST was searched both against GenBank and SWISS-PROT, using BLAST-N and BLAST-X, respectively (Altschul et al. 1990). The best similarities of each EST were systematically analyzed to define the comment lines shown in Figure 3. The criteria used to define whether a sequence is new, identical, or similar to other sequences were arbitrary and served only to give an approximate indication. In general, sequences with a BLAST-N probability $>\mathrm{e}-30$ were considered new sequences. Scores between e- 30 and e- 60 were considered indicative of similarity, whereas scores <e-60 were considered indicative of identity.

\section{ACKNOWLEDGMENTS}

We thank Professor G.A. Danieli and Professor A. Ballabio for critical reading of the manuscript. We also thank Donata Belvini, Vincenzo Favino, Sara Gomirato, Cristina Potrich, Lara Stevanato, Natascia Tiso, and Rosanna Zimbello for assistance in DNA sequencing and Giorgio Rossi for the preparation of acrylamide gels. This work was financed by Telethon Italy (grant B30).

The publication costs of this article were defrayed in part by payment of page charges. This article must therefore be hereby marked "advertisement" in accordance with 18 USC section 1734 solely to indicate this fact.

\section{REFERENCES}

Adams, M.D., J.M. Kelley, J.D. Gocayne, M. Dubnick, M.H. Polymeropoulos, H. Xiao, C.R. Merril, A. Wu, B. Olde, R. Moreno, A.R. Kerlavage, W.R. McCombie, and 


\section{LANFRANCHI ET AL.}

J.C. Venter. 1991. Complementary DNA sequencing: Expressed sequence tags and human genome project. Science 252: 1651-1656.

Adams, M.D., M. Dubnick, A.R. Kerlavage, R. Moreno, J.M. Kelley, T.R. Utterback, J.W. Nagle, C. Fields, and J.C. Venter. 1992. Sequence identification of 2375 human brain genes. Nature 355: 632-634.

Adams, M.D., M.B. Soares, A.R. Kerlavage, C. Fields, and J.C. Venter. 1993a. Rapid cDNA sequencing (expressed sequence tags) from a directionally cloned human infant brain cDNA library. Nature Genet. 4: 373-380.

Adams, M.D., A.R. Kerlavage, C. Fields, and J.C. Venter. 1993b. 3,400 new expressed sequence tags identify diversity of transcripts in human brain. Nature Genet. 4: $256-267$.

Altschul, S.F., W. Gish, W. Miller, E.W. Myers, and D.J. Lipman. 1990. Basic local alignment search tool. J. Mol. Biol. 215: 403-410.

Attardi, G. and G. Schatz. 1988. Biogenesis of mitochondria. Annu. Rev. Cell Biol. 4: 289-333.

Aviv, H. and P. Leder. 1972. Purification of biologically active globin messenger RNA by chromatography on oligothymidilic acid-cellulose. Proc. Natl. Acad. Sci. 69: 1408-1412.

Chomczynski, P. and N. Sacchi. 1987. Single-step method of RNA isolation by acid guanidium thiocyanate-phenol-chloroform extraction. Anal. Biochem. 162: 156-159.

Feinberg, A.P. and B. Vogelstein. 1983. A technique for radiolabeling DNA restriction endonuclease fragment to high specific activity. Anal. Biochem. 132: 6-13.

Frigerio, J.M., P. Berthézène, P. Garrido, E. Ortiz, S. Barthellemy, S. Vasseur, B. Sastre, I. Seleznieff, J.C. Dagorn, and J.L. Iovanna. 1995. Analysis of 2166 clones from a human colorectal cancer cDNA library by partial sequencing. Hum. Mol. Genet. 4: 37-43.

Gieser, L. and A. Swaroop. 1992. Expressed sequence tags and chromosomal localization of cDNA clones from a subtracted retinal pigment epithelium library. Genomics 13: $873-876$.

Höfte, H., T. Desprez, J. Amselem, H. Chiapello, M. Caboche, A. Moisan, M.F. Jourjon, J.L. Charpenteau, P. Berthomieu, D. Guerrier et al. 1993. An inventory of 1,152 expressed sequence tags obtained by partial sequencing of cDNA from Arabidopsis thaliana. Plant $J$. 4: 1051-1061.

Hultman, T., S. Bergh, T. Moks, and M. Uhlén. 1991. Bidirectional solid phase sequencing of in vitro amplified plasmid DNA. BioTechniques 10: 84-93.

Khan, A.S., A.S. Wilcox, M.H. Polymeropoulos, J.A. Hopkins, T.J. Stevens, M. Robinson, A.K. Orpana, and
J.M. Sikela. 1992. Single pass sequencing and physical and genetic mapping of human brain cDNA. Nature Genet. 2: $180-185$.

Ko, M.S.H. 1990. An "equalized cDNA library" by the reassociation of short double-stranded cDNAs. Nucleic Acids Res. 18: 5705-5711.

Liew, C.C., D.M. Hwang, Y.W. Fung, C. Laurenssen, E. Cukerman, S. Tsui, and C.Y. Lee. 1994. A catalogue of genes in the cardiovascular system as identified by expressed sequence tags. Proc. Natl. Acad. Sci.

91: 10645-10649.

McMaster, G.K. and G.G. Carmichael. 1977. Analysis of single-stranded and double-stranded nucleic acids on polyacrylamide and agarose gels by using glyoxal and acridine orange. Proc. Natl. Acad. Sci. 74: 4835-4838.

Okubo, K., N. Hori, R. Matoba, T. Niiyama, A. Fukushima, Y. Kojima, and K. Matsubara. 1992. Large scale cDNA sequencing for analysis of quantitative and qualitative aspects of gene expression. Nature Genet. 2: 173-179.

Patanjali, S.R., S. Parimoo, and S.M. Weissman. 1990. Construction of a uniform-abundance (normalized) cDNA library. Proc Natl. Acad. Sci. 88: 1943-1947.

Pearson, W.R. and D.J. Lipman. 1988. Improved tools for biological sequence comparison. Proc Natl. Acad. Sci. 85: 2444-2448.

Sikela, J.M. and C. Auffrey. 1993. Finding new genes faster than ever. Nature Genet. 3: 189-191.

Takeda, J., H. Yano, S. Eng, Y. Zeng, and G.I. Bell. 1993. A molecular inventory of human pancreatic islets: Sequence analysis of 1000 cDNA clones. Hum. Mol. Genet. 2: 1793-1798.

Thomas, P.S. 1980. Hybridization of denatured RNA and small DNA fragments transferred to nitrocellulose. Proc. Natl. Acad. Sci. 77: 5201-5205.

Wan Kim C., P. Markiewicz, J.J. Lee, C.F. Schierle, and J.H. Miller. 1993. Studies of hyperthermophile Termotoga maritima by random sequencing of cDNA and genomic libraries. J. Mol. Biol. 231: 960-981.

Waterston, R., C. Martin, M. Craxton, C. Huynh, A. Coulson, L. Hillier, R.K. Durban, P. Green, R. Shownkeen, N. Halloran, T. Hawkins, R. Wilson, M. Berks, Z. Du, K. Thomas, and J. Thierry-Mieg. 1992. A survey of expressed genes in Caenorhabditis elegans. Nature Genet. 1: 114-123.

Wilcox, A.S., A.S. Khan, J.A. Hopkins, and J.M. Sikela. 1991. The use of 3' untranslated sequences of human cDNA for rapid chromosome assignment and conversion to STSs: Implication for an expression map of the genome. Nucleic Acids Res. 19: 1837-1843.

Received July 26, 1995; accepted in revised form December 14, 1995. 


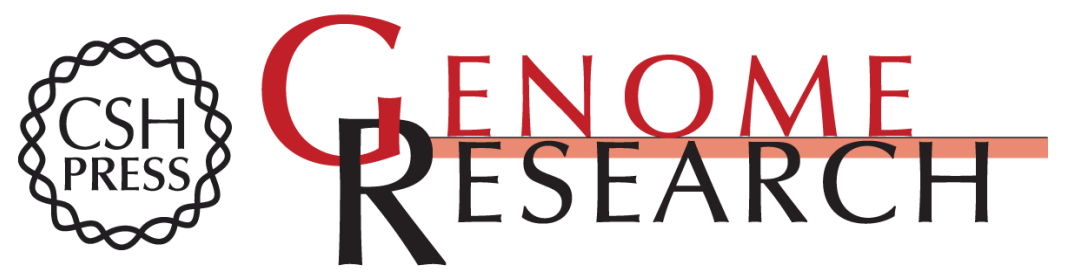

\section{Identification of 4370 expressed sequence tags from a 3 '-end-specific cDNA library of human skeletal muscle by DNA sequencing and filter hybridization.}

G Lanfranchi, T Muraro, F Caldara, et al.

Genome Res. 1996 6: 35-42

Access the most recent version at doi:10.1101/gr.6.1.35

References This article cites 26 articles, 6 of which can be accessed free at: http://genome.cshlp.org/content/6/1/35.full.html\#ref-list-1

License

Email Alerting

Receive free email alerts when new articles cite this article - sign up in the box at the Service top right corner of the article or click here.

\section{Affordable, Accurate Sequencing.}

To subscribe to Genome Research go to: https://genome.cshlp.org/subscriptions 